\title{
PBL e robótica no ensino de conceitos de Lógica de Programação
}

\author{
Flávia Zenaro Nogueira e Silva, Marcos Augusto Francisco Borges
}

\author{
Faculdade de Tecnologia - Unicamp \\ R. Paschoal Marmo, 1888 - 13484-332 - Limeira - SP - Brasil \\ flavia.zenaro@pos.ft.unicamp.br, marcosborges@ft.unicamp.br
}

\begin{abstract}
Studies show that programming logic school subjects have high failure levels, increasing demotivation and dropout of student risks. The use of robotics for educational purposes may help to promote TeachingLearning Processes and knowledge creation improvements by using activities that arouse the interest of students. This paper's goal is to show programming logic concepts teaching proposal using robotics kits as pedagogical tool based on Problem-Based Learning (PBL) method and Collaborative Learning.

Resumo. Estudos apontam que a disciplina de lógica de programação possui altos indices de reprovação, contribuindo para a desmotivação e possível evasão de alunos. $O$ uso de robótica com finalidades pedagógicas pode ajudar a promover situações de ensino-aprendizagem e aumento da construção do conhecimento, pela introdução de atividades que despertam o interesse e aguçam a curiosidade e motivação dos alunos. Este trabalho tem por objetivo apresentar uma proposta de ensino de conceitos de lógica de programação utilizando kit robótico como ferramenta pedagógica baseada no método Problem-Based Learning (PBL) e na Aprendizagem Colaborativa.
\end{abstract}

\section{Introdução}

Os jovens estudantes, fascinados e mergulhados em um mundo de tecnologia e inovação, onde tudo acontece "on-line", requerem um novo tipo de abordagem pedagógica.

O avanço da tecnologia nos permitiu empregar novas ideias na área educacional, como, por exemplo, a utilização da robótica, que é utilizada como ferramenta pedagógica no ensino de disciplinas como lógica e linguagem de programação. $\mathrm{O}$ emprego de computadores e kits de robótica em ambientes educacionais pode ser entendido como um caminho natural, uma ferramenta adequada para o desenvolvimento de atividades que envolvam criar, projetar e planejar, favorecendo assim o processo de ensino-aprendizagem (Chella, 2002).

A disciplina de Lógica de Programação (LP), também conhecida como Algoritmos, está presente em quase todas as grades de cursos de Graduação e Técnico da área de Computação. Essa disciplina também pode ser encontrada em grande parte dos cursos de engenharia, por ajudar a desenvolver o raciocínio lógico e matemático, fator importante para ambas as áreas. Vista por muitos alunos como uma matéria complexa e de difícil entendimento, a disciplina em questão tem papel fundamental para prepará-los nos conceitos básicos de programação para que, posteriormente, possam 
aprender uma linguagem de programação e escrever códigos para computador e dispositivos móveis.

Rapkiewicz et.al, (2006) comentam que a falta de motivação e a dificuldade de desenvolver o raciocínio lógico, necessários para a construção de algoritmos, são os fatores principais da desistência dos ingressantes em programação. Outro fator importante são os altos índices de reprovações nas disciplinas iniciais (Gomes et.al, 2008)(Peixoto et.al, 2013).

O presente artigo propõe formas de trabalhar alguns conceitos de LP utilizando a metodologia PBL (problem-based learning) e kit robótico como ferramenta pedagógica. O restante do artigo está organizado da seguinte forma: a seção 2 apresenta os trabalhos relacionados que contribuíram para o desenvolvimento desta pesquisa, a seção 3 relata o embasamento teórico, a seção 4 descreve a análise dos kits e a proposta de ensino, por fim, a seção 5 discute as considerações finais e os trabalhos futuros.

\section{Trabalhos Relacionados}

Existem várias pesquisas sobre a utilização do kit robótico Lego Mindstorms no ensino, o uso da robótica na educação e ferramentas de apoio para o ensino de LP.

Os autores De Jesus \& Cristaldo (2014) relatam pontos positivos em suas experiências na utilização do Lego Mindstorms em uma olimpíada de robótica para aprendizagem nas áreas de Ciência e Algoritmos com turmas de ensino médio integrado de informática. Constatou-se um aumento no índice das notas acima de $9.0 \mathrm{em}$ aproximadamente $8 \%$ após a participação dos alunos no projeto. Outro fator positivo, observado por esses autores, foi a aceitação do projeto por parte dos alunos, com $80 \%$ das opções de avaliação marcadas como excelente, o que mostra a grande aceitação na participação de atividades com o uso de robótica.

Almeida \& Netto (2015), fizeram uma revisão sistemática na literatura sobre a Robótica no contexto Educacional, Aprendizagem de Programação e Programação de Robôs a Distância. Analisando 82 artigos, segundo esses autores, a robótica no contexto educacional tem sido utilizada de forma interdisciplinar, associada a aplicações desenvolvidas nos kits Lego Mindstorms ou Arduino. Sobre a aprendizagem de programação com robôs, perceberam a utilização de diferentes tecnologias, porém, apenas um trabalho auxiliava no ensino de LP e outros dois faziam uso das linguagens Python e $\mathrm{C}$ para a programação. Chegaram à conclusão que um grande número de métodos e ferramentas vem sendo utilizado como apoio na aprendizagem de programação para robôs.

Neto et. al (2015) também apresentaram uma revisão sistemática do uso da robótica na educação durante os anos de 2004 até 2014. Os autores notaram que a maior parte da pesquisa estava localizada nas regiões Sudeste e Nordeste do país. Identificaram também a Robótica Educacional (RE) como uma matéria interdisciplinar, sendo utilizadas por outras doze disciplinas, como Física e Química, sem contar a própria RE.

A comparação entre as publicações estudadas mostrou que a RE está sendo mais utilizada no ensino fundamental, do que nos ensinos médio, técnico e superior. Outro item importante observado é o grande volume de propostas, principalmente de novos 
produtos de Software, metodologias e robôs para os diversos níveis de ensino, em contra partida, existem poucas experiências práticas relatadas na área.

De maneira geral, os autores tem relatado grande aceitação dos alunos na utilização da robótica como meio de ensinar conceitos de diversas disciplinas. Para a disciplina de LP, no entanto, observou-se a menção de apenas uma aplicação prática. $\mathrm{O}$ presente artigo apresenta sugestões de utilização do ambiente de programação do kit Lego Mindstorms que podem ser usadas em sala de aula para ensinar alguns conceitos de LP.

\section{Embasamento teórico}

\section{1 - Problem-Based Learning (PBL)}

A PBL é uma abordagem para o ensino que tem crescido em todo o mundo desde os anos 70, a qual permite que os estudantes sejam capazes de perceber as situações e estruturas particulares de como eles aprendem e como eles se veem como futuros profissionais (Savin-Baden, 2000).

Segundo Norman e Schmidt (2000), a PBL fornece uma abordagem à educação mais desafiadora, motivadora e interessante. A resolução de problemas reais que podem ser vivenciados no dia-a-dia de uma profissão, faz com que o aluno tenha interação com a vida real, praticando a interação social através da vivência na resolução destes problemas. A PBL também estimula a motivação intrínseca (epistêmica) dos alunos, através da colocação e discussão em sala de aula de problemas relevantes a sua futura área de atuação profissional. Schmidt (2001) afirma em seu trabalho que a motivação epistêmica funciona como uma força interna que leva as pessoas a conhecerem melhor o mundo.

\section{2 - Pensamento Computacional}

O Pensamento Computacional (PC) é o processo de pensamento envolvido na formulação de problemas e das suas soluções, tendo como base os fundamentos da Ciência da Computação. A combinação do pensamento crítico com os fundamentos da computação define uma metodologia para resolver problemas, denominada Pensamento Computacional ou Computational Thinking (Wing, 2006). Define-se como PC "saber usar o computador como um instrumento de aumento do poder cognitivo e operacional humano" (Blikstein, 2008). Esta visão defende que a cultura do computador ajuda a sociedade não somente a aprender, mas especialmente oferece uma nova maneira de aprender a aprender (Lu \& Fletcher, 2009).

PC pode ser considerado, conforme Wing (2006), como uma das habilidades intelectuais básicas de um ser humano, comparada a ler, escrever, falar e fazer operações aritméticas, habilidades estas que servem para descrever e explicar situações complexas. O PC pode ser visto como uma linguagem (como as linguagens escrita e matemática) que pode ser utilizada para as pessoas falarem (descreverem) sobre o Universo e seus processos complexos.

\section{3 - Aprendizagem Colaborativa}

Segundo Torres, Alcantara e Irala (2004, p. 3) a Aprendizagem Colaborativa "é uma estratégia de ensino que encoraja a participação do estudante no processo de aprendizagem e que faz a aprendizagem um processo efetivo e ativo." Ainda segundo os 
autores, os alunos de forma coletiva, constroem seu próprio conhecimento por meio da troca de informações, de pontos de vista, de opiniões divergentes, de resoluções de questões e de avaliações. Outra definição para Aprendizagem Colaborativa é "[...] uma situação em que duas ou mais pessoas aprendem ou tentam aprender alguma coisa juntas” (Dillenbourg, 1999, p. 1, tradução nossa) ${ }^{1}$.

\section{Ferramentas e Métodos}

O presente projeto de pesquisa visa, através do kit robótico escolhido, demonstrar propostas de maneiras para trabalhar alguns conceitos da LP com objetivo de identificar o melhor uso dos recursos oferecidos pelo kit.

O projeto foi dividido em três etapas, sendo elas: análise de kits robóticos disponíveis no mercado, análise dos recursos disponíveis para o kit escolhido e uma formulação de Proposta de Ensino.

\subsection{Análise de kits robóticos disponíveis no mercado}

A primeira etapa consistiu da análise dos kits: Arduino $^{2}$, Lego Mindstorms ${ }^{3}$ e Modelix Robotics ${ }^{4}$.

\section{Arduino}

Foi originalmente desenvolvido como recurso pedagógico para o ensino e depois no ano de 2005 foi lançado comercialmente por Massimo Banzi e David Cuartielles (Monk, 2013).

A placa Arduino pode variar dependendo do modelo escolhido, de maneira geral, é uma plataforma de prototipagem eletrônica de hardware livre e de placa única, projetada com um microcontrolador Atmel AVR com suporte de entrada/saída embutido, o Arduino Software IDE (ambiente de desenvolvimento integrado) pode ser instalado nos sistemas operacionais Windows, Macintosh OSX e Linux, porém, a maioria dos sistemas microcontroladores são limitados para uso no sistema operacional Windows.

Sua linguagem de programação oficial é baseada na linguagem $\mathrm{C} / \mathrm{C}++$, com algumas particularidades para geração automática de protótipos de funções. Os programas escritos usando o Arduino Software IDE são chamados de sketches e salvos com a extensão de arquivo .ino (Arduino, 2016). Um exemplar da placa UNO é ilustrado na Figura 1(a) e do Arduino Software IDE na Figura 1(b).

\footnotetext{
${ }^{1}$ Texto original: "[...] it is a situation in which two or more people learn or attempt to learn something together."

${ }^{2}$ https://www.arduino.cc/

${ }^{3} \mathrm{http}: / /$ www.lego.com/en-us/mindstorms

${ }^{4} \mathrm{http}: / /$ modelix.cc/
} 


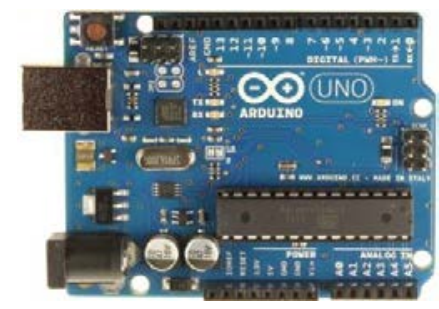

(a) Arduino Uno

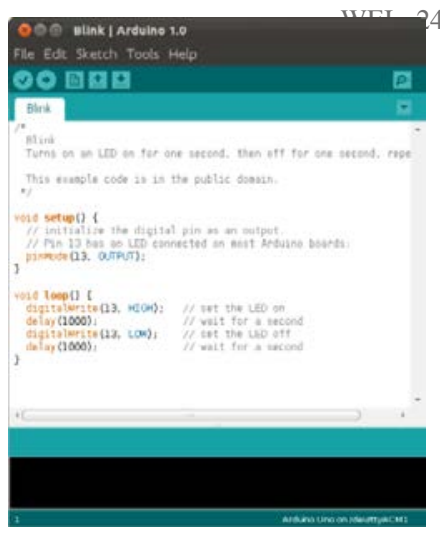

(b) Arduino Software IDE

Figura 1 - Ilustração da placa e do ambiente de desenvolvimento do Arduino. Fonte: https://www.arduino.cc

\section{2 - Lego Mindstorms}

O kit Lego Mindstorms é comercializado pela empresa dinamarquesa LEGO e foi criado em 1980, em parceria com o Instituto de Tecnologia de Massachusetts (MIT) (Lego, 2016).

Sua programação é feita de forma visual através de um ambiente de programação próprio, que pode ser obtido no site oficial da empresa Lego. Sua programação é feita de forma visual, com a utilização de blocos funcionais que estão agrupados em: blocos de ação, blocos de fluxo, blocos de sensores, blocos de operações de dados e blocos avançados (Lego, 2016).

O Brick é a unidade programável do kit onde os programas são carregados, armazenados e executados. Esta unidade contém microprocessador, quatro portas de entrada nomeadas como 1, 23 e 4, quatro portas de saída A, B, C e D, uma porta mini USB, para conectar do Brick a um computador, uma porta para cartão de memória e uma porta USB (Lego, 2016).

O kit possui diversas peças com vários formatos de forma a possibilitar a montagem de estruturas como tanques, carros e robôs ou em formatos como de escorpião e de cobra. Alguns tipos de montagem encontram-se ilustrados na Figura 2.
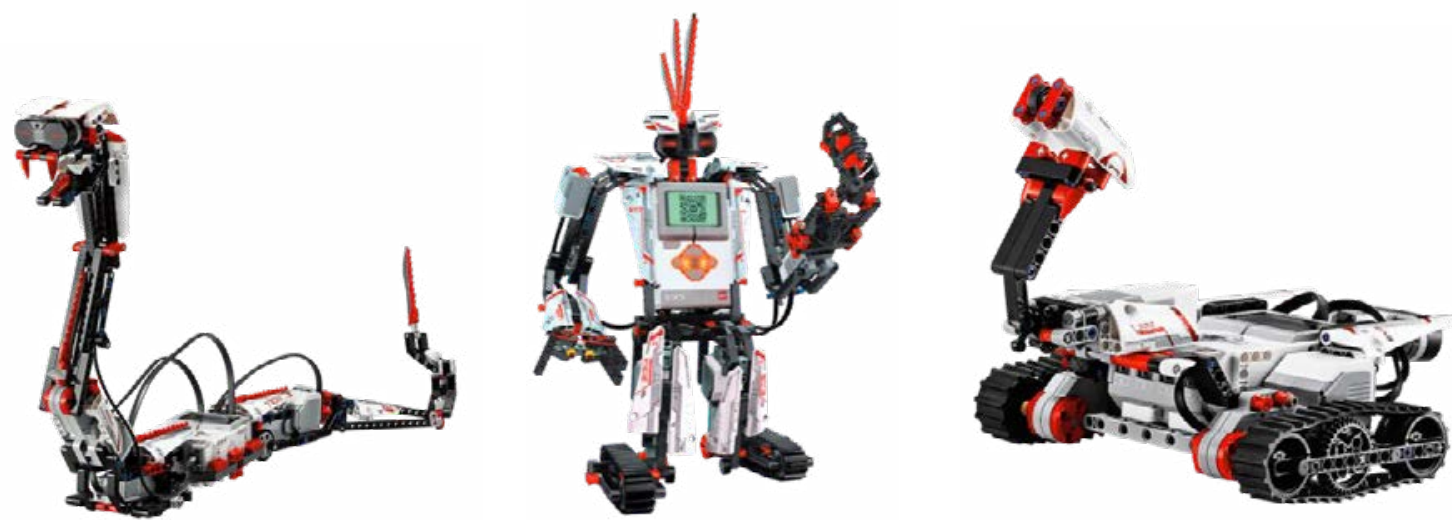

Figura 2 - Opções de montagens com o kit Lego Mindstorms EV3. Fonte: http://www.lego.com/

Atualmente, o kit Lego Mindstorms está em sua terceira geração, chamada de EV3, e traz como inovação a possibilidade dos robôs serem controlados por dispositivos como smartphones e tablets.

3 - Modelix Robotics

O kit robótico Modelix Laboratório de Robótica 411 PLUS é voltado para ensino de robótica com ambiente de programação próprio, onde a programação é feita através 
da construção de fluxogramas, conforme ilustra a Figura 3(a), sem a necessidade de conhecimento de nenhuma linguagem de programação. As peças que compõem o kit permitem alguns tipos específicos de montagem, ilustrados na Figura 3(b). (Modelix, 2016).

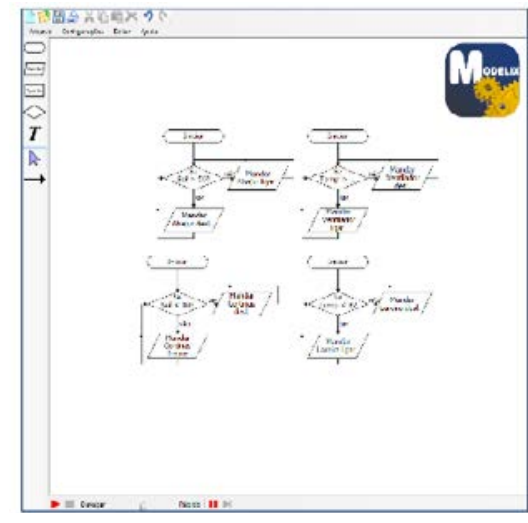

(a) Ambiente de programação

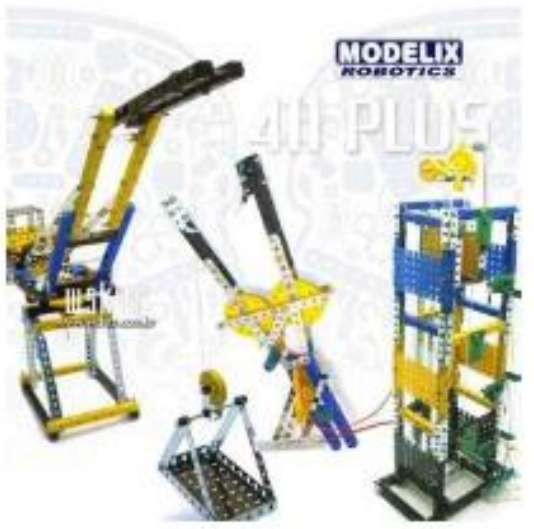

(b) Opções de montagens

Figura 3 - Ambiente de programação e opções de montagens com kit Laboratório de Robótica 411 PLUS - Fonte: http://modelix.cc/

4 - Análise das principais características entre os kits

Na Tabela 1 são listadas algumas características observadas em cada kit as quais são de fator determinante para a escolha do mais apropriado para o ensino de LP.

Tabela 1 - Comparação entre os kits robóticos analisados

\begin{tabular}{|c|c|c|c|c|}
\hline Kits & Montagem & Programação & $\begin{array}{c}\text { Material de } \\
\text { instruções para } \\
\text { montagem }\end{array}$ & Preço \\
\hline Arduino & $\begin{array}{c}\text { É necessário } \\
\text { noções de } \\
\text { eletrônica. Sem } \\
\text { limitação de } \\
\text { opções de } \\
\text { montagem. }\end{array}$ & $\begin{array}{c}\text { Linguagem } \\
\mathrm{C} / \mathrm{C}++\end{array}$ & Ausente & $\mathrm{R} \$ 199,00$ \\
\hline Lego Mindstorms & $\begin{array}{c}\text { Montagem } \\
\text { simples com } \\
\text { peças de encaixe. } \\
\text { Sem limitação de } \\
\text { opções de } \\
\text { montagem. }\end{array}$ & $\begin{array}{c}\text { Ambiente próprio } \\
\text { de programação. } \\
\text { Programação de } \\
\text { modo Visual. }\end{array}$ & $\begin{array}{c}\text { Grande } \\
\text { quantidade de } \\
\text { conteúdo } \\
\text { disponível no site } \\
\text { do fabricante. }\end{array}$ & $\mathrm{R} \$ 2.856,00$ \\
\hline Modelix Robotics & $\begin{array}{l}\text { É necessário } \\
\text { noções de } \\
\text { eletrônica. } \\
\text { Limitação de } \\
\text { opções de } \\
\text { montagem. }\end{array}$ & $\begin{array}{c}\text { Ambiente próprio } \\
\text { de programação. } \\
\text { Uso de } \\
\text { Fluxograma. }\end{array}$ & $\begin{array}{l}\text { Conteúdo } \\
\text { fornecido junto } \\
\text { ao kit. }\end{array}$ & $\mathrm{R} \$ 2.950,00$ \\
\hline
\end{tabular}

Para análise de custo, serão considerados os valores para aquisição de kits de robótica. Para o Arduino, uma opção de kit pode ser encontrada pelo valor de R\$ 
$199,00^{5}$ que vem com o Arduino Uno R3 e com componentes de eletrônica. O kit Lego Mindstorms Education EV3 pode ser adquirido no valor de R\$2.856,00 ${ }^{6}$. Uma opção de kit oferecida pela empresa do Modelix é o kit de robótica 411 PLUS pelo preço de $\mathrm{R} \$ 2.950,00^{7}$. Em questão de custo, a opção do kit Arduino se revela a mais vantajosa em comparação com as demais, no entanto, levando em consideração a usabilidade (facilidade de uso) nos itens de montagem e programação para alunos ingressantes, o kit da empresa LEGO se destaca o mais apropriado como já mencionado anteriormente.

\section{2 - Análise dos recursos disponíveis para o kit escolhido}

Para este projeto, foi escolhido o kit Lego Mindstorms, por oferecer vários tipos de sensores prontos para serem usados como, por exemplo, sensores de toque, luz e presença, como ilustrado na Figura 4, e pelo fato de não precisar de conhecimentos prévios de programação ou do conhecimento de alguma linguagem de programação, como é o caso do Arduino.

Outro ponto positivo para a escolha do kit LEGO, ao invés do kit Arduino e do Modelix, é sua facilidade de montagem, não exigindo conhecimentos de eletrônica, como é caso do Arduino e sendo mais fáceis de encaixar, não necessitando de uso de ferramenta como chave de fenda e o uso de parafusos, como acontece com o Modelix. A não necessidade de conhecimentos de eletrônica é um fator importante, pois a disciplina de LP é oferecida nos semestres iniciais e os alunos, em sua maioria, não possuem conhecimentos avançados de eletrônica. Também existe a facilidade de encontrar exemplos de montagem, tutoriais passo-a-passo e demais materiais de apoio para iniciantes, fornecidos pela própria empresa, através do site da Lego.

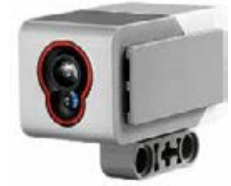

Sensor de cor

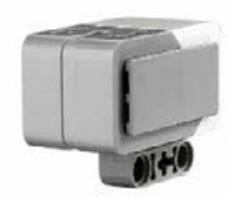

Sensor de rotação

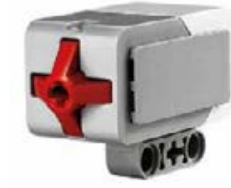

Sensor de toque

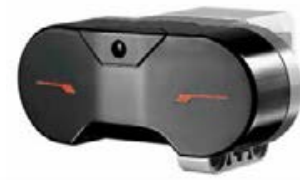

Sensor Infravermelho

Figura 4 - Sensores do kit robótico EV3. Fonte: http://www.lego.com/

\section{3 - Proposta de Ensino}

A proposta está focada em três assuntos da LP: programação sequencial, estrutura de decisão e de repetição.

1. Programação sequencial

\footnotetext{
${ }^{5}$ Arduino Uno R3 Fonte: https://www.robocore.net/modules.php?name=GR_LojaVirtual\&prod=105. Acessado em: 23 de maio de 2016.

${ }^{6}$ Fonte: http://zoom.education/produtos/conjunto-lego-mindstorms-education-ev3. Acesso em: 23 de maio de 2016.

${ }^{7}$ Fonte: http://www.kitsderobotica.com.br/kit-escolar-411-laboratorio-de-robotica. Acesso em: 23 de maio de 2016.
} 


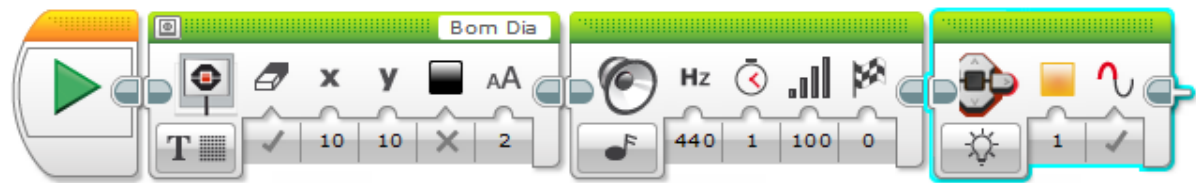

(a)

(b) (c)

Figura 5 - Programação Sequencial. Fonte: do Autor

Para exemplificar uma programação sequencial é proposto o uso dos blocos: Display, Sound e Brick Status Light encontrados na aba Action. A programação proposta está ilustrada na Figura 5.

Desta forma, o aluno pode perceber que o robô executa as ações representadas pelos blocos (a), (b) e (c), ilustrados na Figura 5, de forma sequencial. Ao final da execução do programa, o robô terá feito sequencialmente, a exibição do texto "Bom Dia" na tela do Brick, utilizando o bloco Display, a emissão de um som, através do bloco Sound, e ter acendido a luz amarela do Brick, com o uso do bloco Brick Status Light.

\section{Programação com estrutura de decisão}

Como proposta para utilizar a estrutura de decisão em uma programação, foi criada a programação utilizando o sensor de toque ou Touch Sensor, encontrado na aba Sensor, o qual através de seus estados: 0 - Liberado, 1- Pressionado e 2 Pressionado e Solto, é possível programar uma ação para cada uma das opções de verdadeiro ou falso do estado testado.

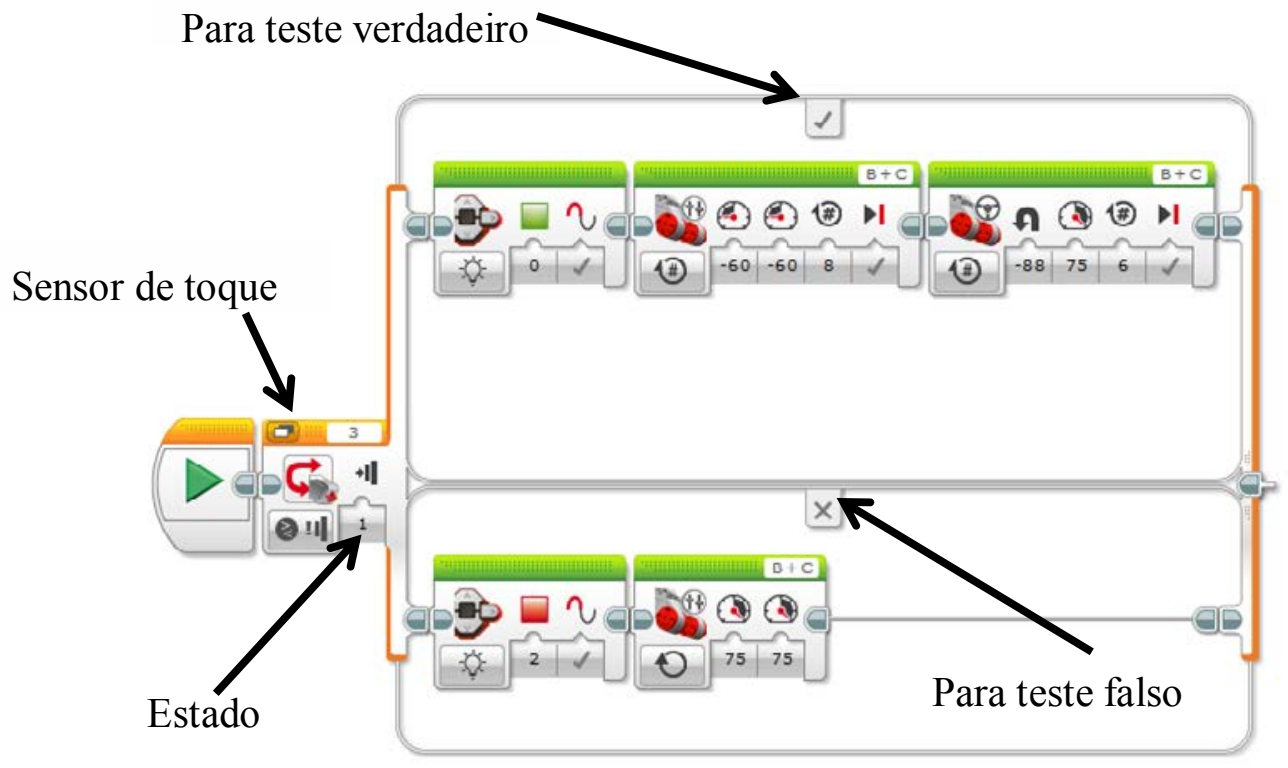

Figura 6 - Programação com Estrutura de Decisão. Fonte: Do autor.

Na Figura 6, a programação pode ser entendida da seguinte maneira: Se o sensor de toque que está com o estado 1, for verdadeiro, que significa que o sensor foi pressionado, então a execução do programa será a da parte superior, fazendo com que acenda uma luz verde do Brick e em seguida o motor gire em sentido anti-horário, fazendo o carro andar de marcha ré e depois faça uma curva. 
Em caso falso, ou seja, se a situação não for de sensor pressionado, a execução executada será a da parte de baixo da imagem, a qual possui um bloco para acender a luz vermelha do Brick e fazer o motor funcionar com rotação para frente.

3 - Programação com estrutura de repetição

Para ilustrar como uma estrutura de repetição pode ser utilizada no ambiente de programação Lego Mindstorms Home Edition, foi proposta a criação da programação ilustrada da Figura 7. Nesta proposta foi utilizado o bloco Loop, encontrado na aba fluxo, o qual tem a função de fazer com que todos os blocos contidos dentro dele sejam repetidos conforme o tipo de repetição escolhida, podendo ser: ilimitada, por um período de tempo, por uma quantidade definida de vezes, entre outras opções.

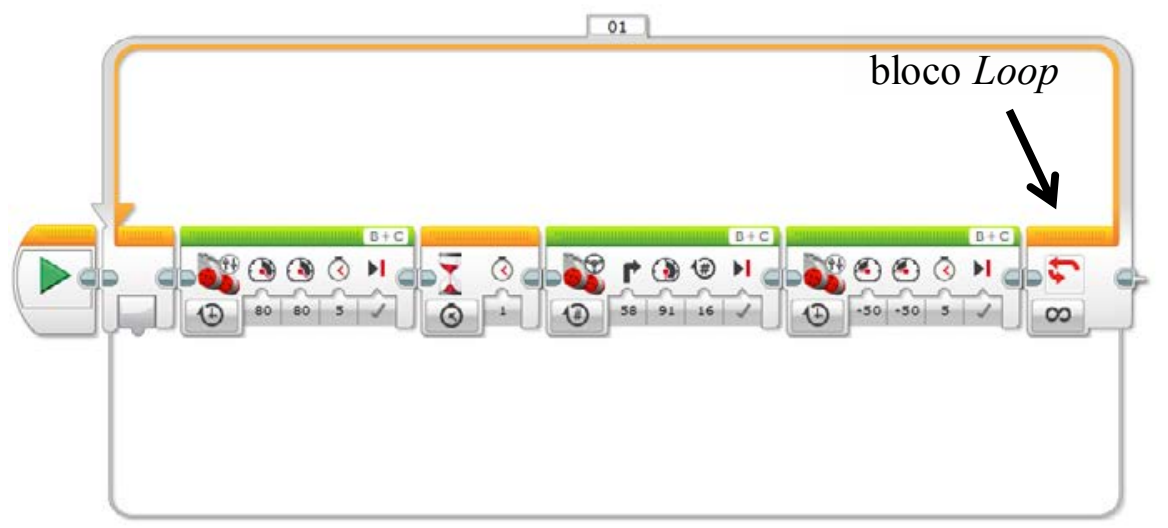

Figura 7 - Proposta de programação com Estrutura de Repetição. Fonte: Do autor.

Com este exemplo pretende-se deixar claro para o aluno o que uma estrutura de repetição faz com a fácil identificação de todos os itens que serão repetidos.

Para aplicação das propostas relatadas neste trabalho é sugerida a divisão dos alunos em grupos, podendo ser de 3 a 7 alunos, de forma a propiciar a Aprendizagem Colaborativa, onde os alunos trabalham de forma conjunta e sem hierarquia definida. Outra sugestão, agora para a aplicação da PBL, seria propor um problema no qual um robô faria o monitoramento e vigilância em uma área determinada. O robô deve percorrer um trajeto, chegando até um ponto definido e retornando ao ponto de partida, contornando possíveis barreiras.

\section{Considerações Finais e Trabalhos Futuros}

Para o ensino de LP é importante atentar para a escolha de um kit de robótica que se encaixe com o perfil dos alunos que farão uso. Para que o objetivo de ensinar LP a alunos iniciantes seja alcançado, a identificação e o uso de estruturas simples e claras são fundamentais para que o assunto trabalhado seja de fácil entendimento.

O presente artigo demonstrou que é possível trabalhar conceitos de LP de uma maneira prática, de forma que possa despertar o interesse dos alunos propiciando a melhor compreensão dos conceitos trabalhados, sem que, para isso, seja necessária a utilização de grande quantidade de recursos do ambiente de programação e tão pouco de recursos complexos.

Como sugestão de trabalhos futuros, podem-se criar atividades de ensino para os conceitos não abordados na proposta apresentada. Além disso, sugere-se a elaboração 
de mais exemplos com a utilização de outros sensores e recursos para os conceitos de lógica descritos deste trabalho.

\section{Referências}

Arduino (2016). Projeto Arduino. http://arduino.cc. Acessado em 21 de fevereiro de 2016.

Almeida, T., Netto, J. F. (2015). Robótica Pedagógica Aplicada ao Ensino de Programação: Uma Revisão Sistemática da Literatura. In Anais do Simpósio Brasileiro de Informática na Educação (SBIE) (Vol. 26, No. 1, p. 597).

Chella, M. T.(2002). Ambiente de Robótica Educacional com Logo. In: XXII Congresso da Sociedade Brasileira de Computação - SBC2002. Florianópolis - SC

De Jesus, L., Cristaldo, M. F. (2014). Uma abordagem utilizando LEGO Mindstorms Education EV3 para verificar o desempenho acadêmico dos estudantes do Instituto, pp. In Anais do Simpósio Brasileiro de Informática na Educação (SBIE) (Vol. 25, No. 1, pp. 1198-1202).

Gomes, A., Areias, C. M., Henrique, J., Mendes, A. (2008). Aprendizagem de programação de computadores: dificuldades e ferramentas de suporte. Revista Portuguesa de Pedagogia, 42, 2, 161-179.

Lego. (2016). LEGO. Acesso em 03 de Outubro de 2015, disponível em The Lego group: http://www.lego.com/en-us/mindstorms/products/ev3/31313-mindstormsev3/

Monk, S. (2013). Programação com Arduino: Começando com Sketches-Série Tekne. AMGH Editora.

Neto, R. P., Santana, A. M., Rocha, D. P., Souza, A. (2015). Robôtica na Educação: Uma Revisão Sistemática dos Últimos 10 Anos. In Anais do Simpósio Brasileiro de Informática na Educação (SBIE) (Vol. 26, No. 1, p. 386).

Norman, G. R.; Schmidt, H. C. (2000). Effectiveness of problem based learning curricula theory, practice and paper darts. Medical Education, 34, 721-728.

Peixoto, M., Scaico, P., Souza, F., Peixoto, H. (2013) Uso de Estratégias de Aprendizagem e Motivacionais pelos Alunos em Disciplinas de Programação: Um Estudo de Caso na Licenciatura em Computação. In: Anais do XXI WEI, p. 460-465. SBC.

Rapkiewicz, C. E. (2006). Estratégicas pedagógicas no ensino de algoritmos e programação associadas ao uso de jogos educacionais. Novas Tecnologias na Educação, 1-2.

Savin-Baden, M. (2000). Problem-Based Learning In Higher Education: Untold Stories: Untold Stories. McGraw-Hill Education (UK).

Torres, P. L.; Alcantara, P. R.; Irala, E. A. F. (2004) Grupos de consenso: uma proposta de aprendizagem colaborativa para o processo de ensino-aprendizagem. Revista Diálogo Educacional. Curitiba, v. 4, n. 13

Wing, J. (2006). Computational thinking. Communications os the ACM Vol 49, 33-35. 